\title{
Bilingualism in Children with a Dual Diagnosis of Down Syndrome and Autism Spectrum Disorder
}

Ward, Rebecca; Sanoudaki, Eirini

\section{Clinical Linguistics and Phonetics}

\author{
Published: 01/07/2021
}

Peer reviewed version

Cyswllt i'r cyhoeddiad / Link to publication

Dyfyniad o'r fersiwn a gyhoeddwyd / Citation for published version (APA):

Ward, R., \& Sanoudaki, E. (2021). Bilingualism in Children with a Dual Diagnosis of Down Syndrome and Autism Spectrum Disorder. Clinical Linguistics and Phonetics, 35(7), 663-689. https://doi.org/10.1080/02699206.2020.1818288

\footnotetext{
Hawliau Cyffredinol / General rights

Copyright and moral rights for the publications made accessible in the public portal are retained by the authors and/or other copyright owners and it is a condition of accessing publications that users recognise and abide by the legal requirements associated with these rights.

- Users may download and print one copy of any publication from the public portal for the purpose of private study or research.

- You may not further distribute the material or use it for any profit-making activity or commercial gain

- You may freely distribute the URL identifying the publication in the public portal ?
}

Take down policy

If you believe that this document breaches copyright please contact us providing details, and we will remove access to the work immediately and investigate your claim. 


\title{
Bilingualism in Children with a Dual Diagnosis of Down Syndrome and Autism Spectrum Disorder
}

\begin{abstract}
Research shows that a substantial proportion of children with Down syndrome (DS) also meet the clinical criteria for Autism Spectrum Disorder (ASD). Children with this dual diagnosis display a linguistic profile that includes significant language delays and language impairments which often differ to the impairments observed in each developmental disability (DD) separately. Given the challenges observed with language acquisition for children with DS-ASD, concerns might be raised regarding the outcomes and suitability of a bilingual environment for children with this dual diagnosis specifically. Limited information is currently available regarding bilingual language outcomes for children with developmental disabilities. Not a single study to date has reported on bilingual language outcomes for children with a dual diagnosis of DS and ASD.
\end{abstract}

The aim of this research was to explore the language profiles of four children with DSASD who had been exposed to two languages. A multiple case-study approach was employed. Four children with a confirmed DS-ASD diagnosis who were aged between six and sixteenyears-old and had received exposure to two languages (English and Welsh) were assessed on a range of cognitive and linguistic measures. Performance was compared to three control groups; bilinguals with DS, aged between six and sixteen-years-old ( $n=10$ ), English monolinguals with DS between five and thirteen-years-old $(n=15)$ and mental age-matched typically developing bilinguals who were aged between three and seven-years-old $(n=25)$. Case studies are presented alongside specialised t-tests modified for analysing deficits in case 
study participants. Assessments comprised of expressive and receptive language, phonological awareness, working memory and non-verbal cognitive abilities.

Considerable variability was found in the cognitive and linguistic profiles of the casestudy participants. Children with DS-ASD displayed similar language profiles to that of the bilingual and monolingual children with DS in the areas tested, although performance was generally lower than that of the TD bilingual children. Similar patterns of weakness were revealed which included marked deficits in expressive language abilities and rhyme awareness. This research documents the bilingual capabilities of children with substantial linguistic and cognitive challenges. Although substantial variability was found, participants were developing bilingual abilities in a similar trajectory to children with DS in line with the degree of exposure to each language. This research highlights the need to assess bilingual children with complex dual diagnoses with an individualistic approach and carefully consider how to appropriately assess and treat bilingual children within speech and language therapy provisions.

\section{Keywords:}

Bilingualism; dual diagnosis; Down syndrome; Autism Spectrum Disorder; language development; language provisions 


\section{Introduction}

Down syndrome (DS) is the most common chromosomal disability, occurring in around 1 in 700-1000 live births (Morris \& Alberman 2009; Parker et al., 2010) and results in moderate to severe intellectual impairments, distinct physical characteristics and a number of complicating health concerns. Alongside these, language impairments are prominent in this population with some aspects of language being disproportionately affected (see Abbeduto, Warren \& Conners, 2007; Ypsilanti \& Grouios, 2008, for a review). Specific weaknesses have been observed in verbal working memory (Lanfranchi et al., 2004), expressive morphosyntax (Andreou \& Katsarou, 2013) and phonological awareness (Kennedy \& Flynn, 2003). Relative strengths have been documented in social abilities, with research suggesting that skills related to social functioning are preserved, which include the ability to orient attention and engage with others (Fidler, 2005).

In contrast, the clinical criteria for Autism Spectrum Disorder (ASD) include "persistent deficits in social communication and social interaction" and "restrictive, repetitive patterns of behaviour" as described in the Diagnostic and Statistical Manual (DSM-5; American Psychiatric Association, 2013). Although language impairments are no longer included in the diagnostic criteria for ASD, difficulties with language including language delays are highly prevalent in this population also, with over half remaining non-verbal or minimally verbal (Tager-Flusberg \& Kasari, 2013). A specific language phenotype is also apparent for this population (Tager-Flusberg \& Caronna, 2007) which includes pragmatic language impairments, echolalia and the use of neologisms (Neely et al., 2016; Parsons et al., 2017). Also in contrast to children with DS, receptive language skills are often on a par with expressive language abilities (Kwok et al., 2015). 
Although these two developmental disabilities (DD) most often occur independently, recent research reports that the occurrence of ASD is considerably higher in those with DS than the general population. Current estimates suggest that between $5-37.7 \%$ of children with DS also meet the diagnostic criteria for ASD (DiGuiseppi et al., 2010; Moss, Howlin, \& Oliver, 2012; Warner et al., 2014) in contrast to estimations from the general population of 1.1-2.24\% (Brugha et al., 2012; Zablotsky et al., 2015). Overlapping symptoms between ASD and DS may make this diagnosis a long and arduous process for these children and their families, which may be further complicated by underlying impairments in hearing and vision (Jensen \& Bulova, 2014). A diagnosis of ASD is not usually advised until children with DS are at least five-years-old (Buckley, 2005). This is not too dissimilar to children without DS as a recent study reported that the average age of diagnosis for ASD is 4;1 (Bent et al., 2015).

More recently, there has been an increase in the accurate identification of children with a dual diagnosis of DS and ASD (henceforth DS-ASD), which has led to an increase in awareness of the behavioural and linguistic phenotype of this dual diagnosis (Warner et al., 2014). Although concerns have been raised about the risk of misdiagnosing ASD in this population due to observed differences in the developmental profiles of children with either ASD or DS (Godfrey et al., 2019), an accurate diagnosis may be particularly imperative and advantageous in adapting speech therapy programmes and educational provisions. Identifying children with DS-ASD will also assist in ensuring that children receive appropriate targeted support in line with expectations for children with this dual diagnosis.

\section{Language and Cognitive Development in DS-ASD}

Research suggests that those with DS-ASD have more impairments than those with singular DS or ASD diagnoses (Godfrey et al., 2019). This profile coincides with some of the 
impairments observed for each DD, however, these impairments in language development appear to be greater for those with DS-ASD, compared to children with DS only. For example, Molloy et al. (2009) found that receptive and expressive language abilities were more impaired in children with DS-ASD compared to children with a single DS diagnosis. They also reported that a number of children with DS-ASD also had a history of a developmental regression in language abilities. Language regressions are typically associated with children with ASD but not children with DS (Barger et al., 2013). Evidence of a language regression in DS-ASD was also reported by Castillo et al. (2008), however, this occurred at a later stage in development for those with this dual diagnosis compared to those with ASD. Furthermore, this regression was also reported to extend to other aspects of development, as opposed to a regression specifically concerning language.

Further research has been conducted by Warner et al. (2014) which explored the impairments that are observed in those with DS-ASD. This large-scale study $(n=499)$ explored autistic tendencies in children with DS, compared to children with ASD. Findings showed that there was a high prevalence of ASD in those with DS as $37.5 \%$ fell within the criteria for ASD, as measured on psychometric evaluations. A number of participants in this study $(16.5 \%)$ met the diagnostic cut-off for autism specifically. Males were more likely to meet the criteria for ASD, with $67 \%$ of those reaching the criteria for ASD being male, a pattern that is similar in children with a single diagnosis of ASD.

Language development appeared to be particularly compromised in the children with DS-ASD with significantly more children from this group at the single word level or below compared to children with DS. In addition, the children with DS-ASD were reported to have a significant delay in their first word of around 6 months. Findings also documented that children with this dual diagnosis were significantly less likely to be able to communicate in 
phases and sentences compared to the children with a single diagnosis of DS. In contrast, the children with DS-ASD displayed fewer impairments than the children with ASD which included measures of social interaction and communication. As a result, the authors suggest that children with a DS-ASD diagnosis should receive assessments and interventions tailored to their needs, which will often differ to that of children with singular diagnoses of DS or ASD.

To date, only one study has reported on intervention outcomes in children with DSASD and this was a single case-study design (Kroeger \& Nelson, 2006). This study aimed to examine the utility of a language programme delivered for one hour a day over a two-week period to a nine-year-old boy diagnosed with DS-ASD. Assessments were conducted prior to the intervention, immediately following the programme and once again at follow-up after nine months post-intervention. Results showed that language production rates increased as a result of the intervention, and importantly, these gains were maintained at follow-up. This study provides promising evidence that interventions with this population specifically can be advantageous and provides a useful starting point in employing a tailored approach according to these children's needs.

\section{Bilingual Language Development in Children with Developmental Disabilities}

Initial investigations have examined language development in children with a number of DD including children with Developmental Language Disorder (DLD; previously referred to as Specific Language impairment or SLI), ASD and DS. Studies in this field now provide increasing evidence that bilingualism does not negatively impact the language development of individuals with DS (Kay-Raining et al., 2005; Feltmate \& Bird 2008; Cleave et al., 2014; Anonymous) ASD (Hambly \& Fombonne, 2012; Valicenti-McDermott et al., 2013; Gonzalez- 
Barrero \& Nadig 2018; Wang et al., 2018) or DLD (Marini et al., 2019; Paradis et al., 2003). Meta-analyses in this field also substantiate the finding that bilingualism is not an aggravating factor in the language impairments that are frequent in these populations (Drysdale, van der Meer, \& Kagohara, 2015; Uljarević, Katsos, Hudry, \& Gibson, 2016).

At the same time, research has reported that parents of children with a DD may be advised to avoid bilingual environments and to abandon native language input if that language differs to the majority community language (Hampton et al., 2017; Howard, Gibson, \& Kastos, 2020; Kay-Raining Bird, Lamond, \& Holden 2012; Yu, 2013). Although more favourable views of bilingualism appear to be emerging in some recent investigations (Marinova-Todd et al., 2016a), findings of studies examining views on the suitability of bilingualism for children with more substantial impairments (such as those with DS-ASD) appear to be less encouraging. Even these recent investigations into professional practices and attitudes towards bilingualism find that clinicians believe that children with more severe language learning difficulties have less of a capacity to be bilingual (Marinova-Todd et al., 2016). These suggestions may lead to parents or caregivers internalising these beliefs that they have to decide between their child developing one single language to varying degrees or acquiring two languages to a less proficient degree.

Opting to remove a native language from the home environment may lead to parents communicating less frequently with the child, using a lower range of vocabulary and inconsistent morphology and less complex grammatical constructions (Altan \& Hoff, 2018). Forced monolingualism in these circumstances may lead to exclusion from opportunities to engage with others and result in less developed social skills (Uljarević et al. 2016). In addition, Kremer-Sadlik (2005) highlighted some of the challenges that arise as a result of limiting language input within bilingual families who had a child with ASD. Restricting language input 
to a single language was reported to place a barrier between the parent and their child as well as other family members. In a recent study, Howard, Katsos and Gibson (2019) suggested that children with ASD themselves believed that bilingualism was a useful resource. This study also reported that a by-product of bilingualism for these children was enhanced emotional and social development, which has substantial implications given the deficits that are usually observed in these aspects for children with ASD specifically.

\section{Bilingual Benefit}

In contrast to this view that bilingualism will further delay or impair language development in children who have a DD, research in the field of bilingualism suggests that there may be cognitive and linguistic advantages, at least for TD children. Studies have reported that bilinguals may display advantages over monolinguals for several abilities which include executive functioning (Carlson \& Meltzoff, 2008), working memory (Morales et al., 2013) and phonological awareness (Bialystok et al., 2003; Canbay, 2011; Chen et al., 2004). Although some have speculated to what extent these benefits are observed under different circumstances (Lehtonen et al., 2018; von Bastian et al., 2016), research suggests that the exposure to two languages accelerates the development of these abilities.

Conversely, children with DS are known to have marked impairments in the development of phonological awareness (Cupples \& lacono, 2000; van Bysterveldt et al., 2006) and working memory (Jarrold et al., 2000). As a result, it is interesting to explore whether there will be additional deficits in their development by the addition of a second language, or whether bilingualism may confer an advantage for this population. Given that phonological awareness is robustly associated with reading, spelling and educational attainment both for TD children (Carnine et al., 2004; Melby-Lervåg et al., 2012) and children 
with DS (Fletcher \& Buckley, 2002), identifying the role that bilingualism has on these aspects of development will provide a novel insight within this population specifically.

\section{Bilingualism in Wales}

Wales provides a natural bilingual platform as the only country in the UK that has two official languages: English and Welsh. Both languages are used within the public domain and according to Welsh language policies, children should be able to access Welsh-medium educational provisions regardless of their home language background. Educational settings in Wales are either Welsh-medium (26.4\%), English-medium (66.1\%), bilingual (5.4\%), or have a dual-stream option meaning that parents are able to opt for Welsh-medium or Englishmedium education (2.1\%; Welsh Government, 2020). As a result, children from all backgrounds are able to access Welsh-medium provisions, regardless of their family's language backgrounds. As the majority language in Wales is English, all speakers of Welsh are naturally exposed to English.

Guidance for children with special educational needs states that bilingualism should be provided "as far as reasonably practicable" (Special Educational Needs Code of Practice for Wales - Paragraph 1.11, 2004). Furthermore, the Welsh government also states in the Welsh Medium Education Strategy (2014) that "no one should be denied opportunities for Welshmedium education or learning Welsh as a language because of their race, ethnicity, disability, gender, sexual orientation, age or religion." Given the policies and provisions that are available in Wales, this natural bilingual platform provides an ideal setting to investigate the bilingual outcomes of children with a DD.

\section{Current Study}


Current understanding of bilingual language development for children with a DD is increasing, however, there still appears to be a lacuna in the area of bilingual language development for children with DS specifically, and even more so in children with a dual DS-ASD diagnosis. To date, no single study has examined the outcomes in children with DS-ASD who have had exposure to two languages. The aim of the present research is to fill this gap by examining bilingualism in children with this dual diagnosis with a specific focus on the aforementioned areas of weakness. This research will examine four unique cases of children with DS-ASD who have received varying exposure to two languages, in comparison to three control groups. In the current study, the following research questions will be addressed:

i. What are the language and cognitive profiles of children with a dual diagnosis of DS and ASD who have been exposed to two languages?

ii. How do the profiles of these children compare to the profiles of bilingual children with DS and typically developing bilingual children?

iii. Do children with a dual diagnosis of DS and ASD who have been exposed to two languages display similar language and cognitive profiles to bilingual and monolingual children with DS?

\section{Method}

A multiple case study design was employed to document the abilities of four unique cases of children who had a diagnosis of DS-ASD or a suspected DS-ASD diagnosis who had been exposed to two languages (specifically Welsh and English). This was deemed the most appropriate method due to the heterogeneous nature of bilingualism and the individual 
differences often observed within these disorders. Each case study is presented descriptively and is also presented in comparison to three control groups. Ethical approval was obtained from the University's departmental ethical review board prior to data collection and informed consent was provided by both parents and schools. Data was stored in line with general data protection regulations which included anonymisation of data and a secure data management process. Assessments were conducted either at home, school, or a combination of the two depending on the suitability of these environments.

\section{Participants}

Welsh-English bilingual and English monolingual children with DS were recruited as part of a larger study on bilingual language development in Wales alongside a bilingual typically developing (BTD) control group (Anonymous). Inclusion criteria stipulated that the children with DS should be aged between five and sixteen-years-old as to recruit a sample of children who were in the process of developing their language skills but had measurable receptive and expressive language abilities. The sample included children who had no more than mild hearing loss, as determined by parental report, so as to include a representative sample of children with DS who often have mild or corrected hearing loss.

Children with DS $(n=32)$ were recruited through contact with two large organisations that support children with intellectual disabilities. During this process, four children with a dual DS-ASD diagnosis were identified, which corresponds to an observed rate of $12.5 \%$ in the current sample of children with DS. Three of these children had a confirmed clinical diagnosis of DS (trisomy 21) and ASD, as confirmed by parental reports. The fourth case study participant had a confirmed diagnosis of mosaic DS and a suspected diagnosis of ASD at the time of data collection, but has since received a confirmed secondary diagnosis of ASD. 
According to NVIQ equivalents on the K-BIT-II, the degree of intellectual impairment varied. Three of the case participants had a severe learning difficulty and the final participant had a NVIQ that was within the average range for her chronological age.

The BTD group was recruited to enable a comparison of typical bilingual development alongside bilingual development in children with DS (BDS). Typically developing children were recruited through contact with local schools and nurseries with children of the specified age range of three and seven-years-old. This range was selected as it was anticipated that children between these ages would be comparable in terms of cognitive abilities to the children with DS. The inclusion criteria for the BTD children specified that they should not have any known or suspected language or intellectual impairment.

Bilingual status was confirmed by parental reports of current and lifetime exposure to Welsh and English. The parental questionnaire also required parents to specify the percentage of time that their child responded in each language, the age of exposure to each language, the consistency of exposure and details pertaining to home language use. Statistical analyses confirmed the group status of bilinguals and monolinguals with the bilingual groups (BTD and BDS) receiving significantly more exposure to Welsh than the monolinguals with Down syndrome (MDS; $p<.05$ ). Bilingual groups were also statistically matched on the age of exposure to a second language $(p=.983)$.

Three children with a dual DS-ASD diagnosis had been continually exposed to Welsh and English. The final case study presented documents a case whereby bilingual exposure was received from birth, however, after the age of five, only English was spoken as the result of receiving advice that this would be beneficial to her linguistic development. This case was included as to explore the impact of early bilingual exposure on language and cognitive development. This case also provides a unique opportunity to document whether the removal 
of one language would have any impact on language outcomes. The demographic information relating to each case study participant are presented in Table 1. Case descriptions containing further information about each case study participant and control groups are presented below.

[Insert Table 1 about here]

\section{Case Description 1: Dylan}

Dylan was aged 10;5 and displayed evidence of a severe learning difficulty as measured by the non-verbal cognitive assessment, with a developmental age of below four years. He was attending a mainstream Welsh-medium primary school and was supported by a one-to-one teaching assistant. Dylan was exposed to both Welsh and English from birth and he had been continuously exposed to both languages. Parent reports confirmed that he was Welsh dominant with current and lifetime input to Welsh at $80 \%$.

\section{Case Description 2: Catrin}

At the time of data collection, Catrin was aged 13;3 and had a confirmed diagnosis of both DS and ASD. The non-verbal cognitive assessment also placed her within the severe learning difficulty category with a developmental age below four years. Until the age of around fiveyears-old, she had received input to both Welsh and English, however, after this stage a decision was made to place Catrin within an English only environment. After this stage, she was attending an English-medium school and only received exposure to English. As a result, Catrin was only assessed in English. Her lifetime exposure to Welsh was reported to be $20 \%$, according to parent report estimates. 


\section{Case Description 3: Owain}

The oldest participant at data collection was Owain who was aged 16;8, however, he also had a developmental age of below four-years-old and the non-verbal cognitive assessments defined him as having a severe learning difficulty. Owain had a confirmed diagnosis of DS and ASD alongside ADHD and parental reports confirmed that he was Welsh dominant with a lifetime exposure to Welsh at $80 \%$. He was attending a resource unit within a Welsh-medium mainstream secondary school and had been consistently exposed to both Welsh and English since birth.

\section{Case Description 4: Rhiannon}

The final case study participant had a suspected ASD diagnosis at the time of testing but has since received a confirmed diagnosis. Rhiannon also had a confirmed mosaic DS diagnosis and was aged 6;8. Non-verbal cognitive assessments showed that she had a developmental age which was almost commensurate to her chronological age at 6;3. Rhiannon was attending a very small Welsh-medium mainstream school with only three other pupils in her class. As with the other participants, she received exposure to both languages from birth and had been consistently exposed to both, however, she was reported to be English dominant with parental reports stating that she had a current and lifetime exposure to Welsh at $20 \%$.

\section{Control Groups}

Three control groups were recruited alongside the case study participants which were: WelshEnglish bilingual children with DS (BDS; $n=10)$, Welsh-English bilingual typically developing children (BTD; $n=25)$ and English monolingual children with DS (MDS; $n=15$ ). Table 2 
provides a summary of the descriptive information pertaining to the three control groups. All parents confirmed that the control group participants had no diagnosed or suspected diagnosis of ASD, or any DD or language impairment for the TD group. Control participants were recruited from similar geographical locations in Wales as the case study participants. All control groups were matched for non-verbal mental age (NVMA) to the BDS group $(p=1.000)$. This resulted in the BTD group being significantly younger than the DS groups $(p<0.001)$. Statistical analyses confirmed no significant differences for chronological age between the two DS control groups $(p=1.000)$. Further statistical analyses confirmed that the bilingual children received significantly more exposure to a second language $(p<.001)$. No significant differences were found for parental socioeconomic status $(p=.85)$ or gender $(p=.88)$ between control groups.

[Insert Table 2 about here]

\section{Materials}

Tasks were selected with the intention of targeting skills that are often impaired in children with DS (i.e. expressive morphosyntax, phonological awareness and working memory). Children were assessed on a range of standardised language and cognitive assessments alongside bespoke linguistic tasks. Assessments in both English and Welsh were undertaken where possible in order to provide a comprehensive overview of language abilities. In order to assess NVMA, the matrices subtest of the Kaufman's Brief Intelligence Test (KBIT-II; Kaufman \& Kaufman, 2004) was utilised. Assessments were also undertaken of working memory by means of a forward digit span task. 
For English language abilities, the seven primary subtests of the Clinical Evaluation of Language Fundamentals - Preschool Second UK Edition (CELF-P; Wig et al., 2006) were administered to provide a comprehensive overview of language abilities. A subset of the Prawf Geirfa Cymraeg: Fersiwn 7-11 (The Welsh Vocabulary Test: Version 7-11; Gathercole \& Thomas, 2007) was employed, testing Welsh receptive vocabulary; this test is the only standardised Welsh language assessment currently available.

Assessments of phonological awareness (PA) were administered in English and Welsh. As there is no standardised assessment for this in Welsh, six bespoke tasks were designed with equivalent tasks in English that were designed to be of equal difficulty to the Welsh assessments. These comprised of two assessments for each component of PA (rhyme/onset, syllable and phoneme) with one implicit and one explicit awareness task for each. The six tasks were: rhyme identification, rhyme generation, syllable segmentation, syllable deletion, initial phoneme matching and phoneme segmenting. The stimuli for Welsh and English task did not differ in terms of word frequencies $(p=.143)$, imageability $(p=.103)$, number of syllables $(p=.143)$ or the number of letters $(p=.410)$.

In order to collect detailed information relating to the children, their language backgrounds and their parents' background, a questionnaire was administered to parents prior to data collection. The parental questionnaire was provided to parents during initial meetings with parents, or was returned via schools. Information provided in the questionnaire also enabled calculations of parental socioeconomic status (SES) using composite scores of parental occupation and education level. For further information on the tasks and materials, see (Anonymous).

\section{Procedure}


All participants were assessed in a one-to-one setting with the first author on the battery of cognitive and linguistic assessments described above. The English language assessments were conducted with all participants and the Welsh language assessments were conducted for all children who were currently receiving Welsh language exposure. Assessments were conducted in a predefined order which was identical for all participants as presented in Table

3. All children in the bilingual groups completed the English and Welsh assessments. Three of the four case study participants still received ongoing exposure to both languages and completed all assessments (except Catrin who had been receiving exposure to English only after the age of 5).

[Insert Table 3 about here]

\section{Data analysis}

As the children with DS were outside of the targeted age ranges for the standardised assessments, raw scores are reported and were used for all data analyses. Statistical analysis was employed to compare the performance of each of the case study participants in comparison to each control group. In order to undertake these analyses, the Revised Standardized Difference Test (RSDT; Crawford \& Garthwaite, 2005; Crawford \& Garthwaite, 2007), was utilised. As NVMA varied across case participants and control groups, analyses on the language and phonological awareness data were undertaken with raw scores on the KBIT as a covariate. The RSDT is a modified t-test that was developed for analysing single-case study data in comparison to a control group to identify any deficits in the case study participant's performance. This enabled statistical analyses to be undertaken in order to ascertain if there were any significant differences in the language and cognitive abilities 
between the case study participants and the control groups. This test was developed to enable the analysis of neuropsychological case study research which also provides point and interval estimates of effect sizes, controls for type 1 errors regardless of small sample sizes and is also robust with skewed data (Crawford \& Howell, 1998; Crawford et al., 2003).

\section{Results}

The results for each case study participant are presented individually below. For detailed comparisons between the control groups, the reader is referred to (Anonymous).

\section{Dylan}

No significant difference was found in terms of non-verbal cognitive ability between Dylan and the control groups. Dylan had an age of first word that was significantly earlier than the BDS control group $(p<.001)$ but not significantly different from the two other control groups (MDS, $p=.415$, BTD, $p=.930$ ). For all assessments on the CELF-P, Dylan performed comparably to all groups, except for the basic concepts subtest where he performed significantly lower than all groups $(p<.05)$. No significant differences were revealed for his performance on the Welsh receptive vocabulary assessment compared to controls. Phonological awareness was also comparable to all groups in English, with the exception of overall PA, whereby Dylan performed significantly higher than the BTD group. Phonological awareness in Welsh was significantly higher than the BTD group for rhyme, phoneme and overall phonological awareness and higher than the BDS group for rhyme. His working memory was significantly higher than all control groups. Dylan's results are summarised in Table 4. 
[Insert Table 4 about here]

\section{Catrin}

Catrin did not perform differently to the control groups for her non-verbal cognitive ability. Her age of first word was significantly later than all of the control groups (at 48 months; BDS, $p<.001$, MDS, $p<.05$ and BTD, $p<.001)$. Statistical analyses revealed no significant differences on the English language assessments between Catrin and any control group. Her performance was significantly higher than the BTD group for overall phonological awareness $(p=.04)$, and higher than the BDS $(p=.01)$ and BTD $(p=.01)$ groups for rhyme-level tasks. No significant effect was found for working memory. Catrin's results are summarised in Table 5.

[Insert Table 5 about here]

\section{Owain}

Owain had the lowest non-verbal cognitive ability and this was significantly lower than the BTD control group $(p<.05)$. His age of first word was not significantly different to the BDS ( $p$ $=.727)$ and $\operatorname{MDS}(p=.845)$ control groups but significantly later than the BTD group $(p<.01)$. A significant effect was found for Owain's performance on the basic concepts subtest where he performed lower than the BTD group only $(p<.001)$. Owain performed significantly higher than the BTD group for overall English phonological awareness $(p=.03)$, and higher than the BTD group for rhyme $(p=.04)$, phoneme $(p=.01)$ and overall phonological awareness $(p=$ .02) in Welsh. No significant effect was found for working memory. Owain's results are summarised in Table 6. 
[Insert Table 6 about here]

\section{Rhiannon}

Rhiannon's non-verbal cognitive ability did not significantly differ from any of the control groups. Her age of first word was not significantly different to the MDS $(p=.571)$ and BTD ( $p$ $=.403)$ group, but was significantly earlier than the BDS group $(p<.01)$. Although Rhiannon had a similar developmental age to all control groups, she performed significantly lower than the BTD group on the sentence structure $(p=.03)$, recalling sentences $(p=.01)$ and concepts and following directions $(p=.03)$ subtests. No significant effect was found on the English language measures compared to DS control groups. Her Welsh receptive vocabulary was significantly lower than both bilingual groups. Statistical analyses found no differences between Rhiannon and the DS groups for phonological awareness in Welsh except for the syllable-level tasks $(p=.01)$. She performed significantly lower than the BTD group for rhyme $(p<.001)$, syllable $(p=.04)$ and overall phonological awareness $(p=.02)$ in English and significantly lower than the BTD group for rhyme $(p=.01)$ and syllable-level tasks $(p=.01)$ in Welsh. No significant effect was found on working memory between Rhiannon and any control group. Rhiannon's results are summarised in Table 7.

[Insert Table 7 about here]

\section{Result Summary}


In summary, no significant differences were found between the children with DS-ASD and the bilingual and monolingual control groups of children with DS for any of the English language assessments. The only exception to this was Dylan who had a lower performance on one subtest (basic concepts). For Welsh receptive vocabulary, only one case participant performed below that of the bilingual control groups (Rhiannon). Comparisons with the typically developing bilingual children found mixed results, with case participants performing significantly lower on several measures. For the phonological awareness assessments, three of the case study participants performed significantly higher for at least one task compared to the BTD group and Catrin performed significantly higher than all control groups. Rhiannon performed similarly to the DS control groups for phonological awareness, but lower than the BTD group. Working memory was comparable between control groups and all case study participants, with the exception of Dylan who performed significantly higher than all control groups.

\section{Discussion}

This multiple case-study documented four unique cases of children with dual diagnoses of DS and ASD who had received exposure to two languages. These were compared to three control groups: Welsh-English bilingual children with DS, English monolingual children with DS and Welsh-English TD bilingual children. Four children from the sample of children with DS were identified as having a confirmed additional diagnosis of ASD. This corresponds to an observed rate of $12.5 \%$ of children in the current sample who were recruited as part of a larger study on language development in children with DS (Anonymous). Previous research estimates that prevalence of ASD in children with DS is around $16-18 \%$ when measured on a standardised 
checklist for autism or by using a social communication questionnaire (DiGuiseppi et al., 2010; Richards et al., 2015). The observed rate in the current sample is in line with reports that ASD prevalence is considerably higher than in the general population (1.1\%-2.24\%; Brugha et al., 2012; Zablotsky et al., 2015).

In considering the age of first word for the case study participants, this milestone was reached within a large range between the four case study children. Two had an age of first word that was significantly earlier than the BDS group (Dylan and Rhiannon) who were reported to have an age of first word of 12 and 15 months respectively. In contrast, one case study participant (Catrin) had a substantial delay in her first word, where this was reported to be at 48 months of age, which was significantly later than all control groups. The final participant (Owain) had an age of first word that was comparable to the DS control groups (24 months), but significantly later than the TD control group. Although it appears that these profiles are similar to reports that children with DS-ASD have further delays in language onset than children with DS (Warner et al., 2014), this was only the case for two of the case study children in this study, which highlights the variability seen in this population. Further research is needed to examine the role of bilingualism on early language milestones in children with DS and children with more complex language impairments such as those with DS-ASD.

Findings show that for the most part, children with DS-ASD performed similarly to monolinguals and bilinguals with DS on measures of receptive and expressive language. Deficits were found between the case study participants and the younger TD children, even after statistically controlling for NVMA. This was particularly true for the English language assessments, and interestingly, was most evident for the participant with mosaic DS. This suggests that bilingual children with DS-ASD were developing language abilities in line with expectations for monolingual and bilingual children with DS. This means that not only is it 
possible for children with DS-ASD to develop language abilities in two languages, but that bilingualism does not appear to further delay or impair language outcomes, as has also been reported in children with singular DS (Kay-Raining et al., 2005; Feltmate \& Bird 2008; Cleave et al., 2014; Anonymous) or ASD diagnoses (Hambly \& Fombonne, 2012; Valicenti-McDermott et al., 2013; Gonzalez-Barrero \& Nadig 2018; Wang et al., 2018).

Molloy et al. (2009) found that children with DS-ASD had more impairments in their receptive and expressive language abilities compared to children with a single DS diagnosis. Similarly, Warner and colleagues (2014) documented that children with both DS and ASD were significantly more likely to be at the single word level and less likely to be able to communicate in phrases or sentences. In the current study, the finding that the children with DS-ASD performed comparably to the children with DS on receptive and expressive language in English and receptive vocabulary in Welsh is in contrast to these previous reports. This contrast may reflect the different methods employed as the present research used a multiplecase study approach as opposed to a group analysis. At the same time, the large range that was found, which is often characteristic of both children with DS and ASD, may explain why these effects did not emerge. This justifies the case-by-case approach taken as the children with DS-ASD presented here had varying abilities from those with fairly substantial cognitive and linguistic impairments to those with fairly preserved cognitive abilities, as seen in the case of the participant with mosaic DS. Alternatively, it could be speculated that exposure to two languages facilitated the development of their linguistic abilities which may explain why the DS-ASD participants did not perform significantly lower than the DS groups. Future studies employing group designs alongside a monolingual DS-ASD group are needed to further explore the impact of bilingualism for this population. 
For the case study participant who had mosaic DS, although her language and phonological awareness abilities were lower than the TD children, her NVMA was equivalent to $6 ; 3$, which was not significantly different to all control groups and was only slightly below her chronological age $(6 ; 8)$. This would be in line with research that shows that children with mosaic DS usually do not experience cognitive impairments to the same extent as children with trisomy 21 (Korbel et al., 2009; Papavassiliou et al., 2015; Zhao et al., 2015). Furthermore, the degree of mosaicism (i.e. the number of cells containing the additional copy of the $21^{\text {st }}$ chromosome) has also been found to correlate with the severity of cognitive impairment (Papavassiliou et al., 2015). As a result, the contrast between her cognitive and linguistic abilities highlights the disparity that exists between these abilities for children with DS, and coincides with reports that children with DS display specific difficulties with language even after considering NVMA (Caselli et al., 2008; Martin et al., 2009).

The profiles found in each of the children's languages also correspond to the dominant language of the case study participants. Rhiannon performed lower than both bilingual groups for the Welsh receptive vocabulary assessment, which reflects the fact that she was English dominant. In contrast, Dylan and Owain performed below the BTD group for one of the English language subtests, but not for the Welsh language assessment. Considering that they were both Welsh dominant according to parental reports, the abilities observed for the children with DS-ASD reflected the amount of input that each child had received in their languages.

Prior research has suggested that bilingualism may enhance several distinct abilities which include phonological awareness (Bialystok, Majumder \& Martin, 2003; Verhoeven, 2007) and working memory (Morales et al., 2013), although some have raised caution with generalising these findings to all bilingual circumstances (de Bruin et al., 2015; Lehtonen et 
al., 2018). Although performance of the bilingual control groups did not differ from the MDS group (see Anonymous), three of the case study participants with DS-ASD performed significantly higher than the BTD group for phonological awareness, and Dylan performed significantly higher than all control groups on the working memory measure. While it is tempting to speculate whether bilingualism conferred an advantage for this population, as these effects were also found compared to the bilingual control groups, it is likely that these findings are not due to bilingualism. As the children with DS were older than the BTD children, they would have received more exposure to literacy instruction, which is a plausible explanation for these findings.

In summary, these findings highlight that children with DS-ASD who have been placed in bilingual environments display the expected profiles of cognitive and linguistic development for children with this dual diagnosis. Although there was considerable variability observed in language and cognitive abilities, the children with DS-ASD who continued to be exposed to two languages did not show any greater language impairments than the DS control groups. Deficits were observed in comparison to the TD control group which evidences the linguistic impairments that are found for children with DS and DS-ASD, even after considering NVMA (Chapman \& Hesketh, 2000; Chapman, Seung, Schwartz, \& Bird, 1998; Warner et al., 2014). This preliminary study suggests that the literature which documents no additional adversity as a result of bilingualism for those with either DS or ASD can be applied to children with DS-ASD when considered on a case-by-case basis. The language abilities found were commensurate with the degree of exposure to each language and in line with expectations for children with DS only, after considering non-verbal cognitive abilities. 


\section{Study limitations}

The findings reported in this multiple case-study should be considered as preliminary and caution is needed in interpreting the findings due to the heterogeneous nature of linguistic abilities in these populations and the small sample size (which is often characteristic of research within this field). Larger samples and group design studies will be advantageous to substantiate the findings reported here, however, the aim of this study was to compare the four unique profiles of children with DS-ASD with control groups of children with DS only and TD children. Although single case study comparisons with control groups may be less likely to find significant results, this approach was taken to compare and contrast these language and cognitive profiles due to the varying profiles in both DS and ASD and gain an insight into the uniqueness of the cases studied. Larger group design studies are likely to mask these often large within-group variability that is found in children with DS and children with DS-ASD to an even greater extent. The variability that was observed between the case studies justified the case by case approach taken for this study. Given that bilingualism is also a highly variable concept with a range of variables to consider, case analyses such as these can provide valuable insights into these fairly unique circumstances.

The second limitation to consider is that this study was conducted as part of a larger study on bilingualism in children with DS (Anonymous). As a result, the tasks and assessments employed were selected in line with the linguistic difficulties that are observed in children with DS specifically, not ASD. Although this study has provided a novel and substantial insight into bilingual profiles of children with DS-ASD, future studies should endeavour to target specific weaknesses associated with the linguistic profiles of children with ASD also, for example, the pragmatic deficits often observed (Loveland et al., 1988; Parsons et al., 2017). Future research that investigates how these areas are impacted by bilingualism will provide 
further information on the linguistic development of children with DS-ASD who receive exposure to two languages. The present study is the first to document bilingualism in children with a dual diagnosis of DS-ASD and consequently, further research in this population specifically is required.

Relatedly, some of the assessments that were employed in this study are not standardised for use with children who have disabilities. There remains a paucity of assessments that are standardised for use with children who have a DD, particularly in terms of measures that adequately assess phonological awareness. In bilingualism research, this becomes more problematic as equivalent assessments do not usually exist across languages, particularly for minority languages, such as Welsh. As a result, bespoke phonological assessments were designed for this research specifically which were tailored towards the populations and languages under study. In addition, the CELF-P was utilised to assess language abilities in English and this assessment is designed for use with clinical populations, and has a high internal consistency for these groups of children.

\section{Research Implications}

There are a number of implications arising from this research that relate to clinical practice. This is the first piece of research to document the bilingual capacities of children with a dual diagnosis of DS-ASD. As a result, these findings have advanced understanding of bilingual development in children with substantial and complex impairments. Previously, it has not been clear how children with this specific dual diagnosis would be impacted by bilingualism, with reports suggesting that practitioners and educators have substantial concerns regarding bilingualism for children with severe language delays and impairments (Ware et al., 2015; Marinova-Todd et al., 2016). This novel study has evidenced that even children with this dual diagnosis and substantial cognitive 
and linguistic impairments can develop language skills in two languages with no evidence to suggest that they experience any additional difficulties compared to bilinguals and monolinguals with a single DS diagnosis.

Findings of this research highlight the need to consider each child on a case-by-case basis and ensure that families and educators receive evidence-informed guidance so that each family can make an informed decision regarding language use as opposed to assumptions that children with complex DDs will not be able to develop skills in two languages. At the same time, timely assessments that are appropriate for bilingual children with complex DDs are needed. Converging evidence is emerging which documents that bilingualism does not negatively impact on the language development of children with DS (Anonymous; Kay-Raining Bird et al., 2005; Feltmate \& Kay-Raining Bird, 2008; Cleave et al., 2014) ASD (Hambly \& Fombonne, 2012; Valicenti-McDermott et al., 2013; Gonzalez-Barrero \& Nadig, 2018; Wang et al., 2018) or DLD (Marini et al., 2019; Paradis et al., 2003). These preliminary findings suggest that children with more severe language and communication needs can also succeed within bilingual environments if given adequate support and opportunities.

Furthermore, policies and guidelines are needed to reflect findings in the field of bilingualism for children with developmental disabilities to ensure that children receive assessments and interventions that are in line with their linguistic needs. Clear guidelines will enable clinicians to provide evidence-based information to families and employ clinical practice that reflects up-to-date research findings in the field. Positive changes have been made in line with research to date with recent guidelines provided by the RCSLT stating that "parent(s) and carers should never be advised to abandon home language in favour of the majority language (typically English, Welsh or Gaelic) under any circumstances. Such an approach is not supported by the evidence base and leads to poor speech, language and 
communication outcomes for the child, and poor social outcomes for the child, family and wider community" (Bilingualism-Guidance Key Points: RCSLT, 2019).

\section{Conclusion}

This is the first study to document bilingualism in children with DS-ASD to date, showing that bilingualism in this population is possible. The aim of this research was to explore the language and cognitive profiles of four children dually diagnosed with DS-ASD who had varying exposure to Welsh and English. This novel study has provided a new understanding of bilingual language development in an under-researched population who often display substantial impairments in cognitive and linguistic abilities. Findings show that these children had varied but considerable impairments in relation to their cognitive, language, working memory and phonological awareness abilities, which is in line with research that shows that children with a dual DS-ASD diagnosis exhibit considerable challenges within these domains. Bilingualism did not appear to have any negative impact on these abilities compared to DS controls. In English, only one participant with DS-ASD performed lower than both DS control groups and this was only for one (out of six) subtest, which was likely to be a reflection of the fact that he was Welsh dominant. All children appeared to be developing language abilities in line with expectations for children with DS only, after considering language input and nonverbal cognitive abilities.

The case studies presented here may assist in understanding how bilingualism impacts development in these children and that language input may play an important role in children with DS-ASD. This factor should be carefully considered when an additional diagnosis of ASD is made, ensuring that full assessments of behaviour, social skills and language are conducted in all languages children are exposed to. Further research is needed to better understand this 
dual diagnosis and to identify effective ways to support linguistic development. This will assist in ensuring that children are supported in the best way possible to enable optimal cognitive and linguistic outcomes.

\section{Acknowledgements}

The authors are very grateful to all the children, schools and families that participated in this research. We are also very grateful to the Down's Syndrome Association UK for collaborating and promoting this research with their networks. We thank the two anonymous reviewers for the valuable feedback provided in reviewing an earlier version of this paper.

Declaration of Interest: The authors report no conflicts of interest. The authors alone are responsible for the content and writing of the paper.

\section{Funding}

This work was supported by a Wales ESRC-DTP studentship secured by the second author in collaboration with the UK Down's Syndrome Association and awarded to the first author, and supported financially by [Anonymised for review].

Word Count: 8,289 


\section{References}

Abbeduto, L., Warren, S. F., \& Conners, F. A. (2007). Language development in down syndrome: From the prelinguistic period to the acquisition of literacy. In Mental Retardation and Developmental Disabilities Research Reviews, 13(3), 247-61. https://doi.org/10.1002/mrdd.20158

Altan, A., \& Hoff, E. (2018). Complex structures in the child-directed speech of native and nonnative speakers. In Psycholinguistics and Cognition in Language Processing. https://doi.org/10.4018/978-1-5225-4009-0.ch007

Andreou, G., \& Katsarou, D. (2013). Language Learning in Children with Down Syndrome (DS): Receptive and Expressive Morphosyntactic Abilities. Procedia - Social and Behavioral Sciences, 93, 921-24, https://doi.org/10.1016/j.sbspro.2013.09.304

Americal Psychological Association, (2013). Diagnostic and statistical manual of mental disorders : DSM-5. In American Psychiatric Association. Diagnostic and statistical manual of mental disorders, Fifth edition.

Barger, B. D., Campbell, J. M., \& McDonough, J. D. (2013). Prevalence and onset of regression within autism spectrum disorders: A meta-analytic review. In Journal of Autism and Developmental Disorders, 43, 817-28. https://doi.org/10.1007/s10803-0121621-x

Bent, C. A., Dissanayake, C., \& Barbaro, J. (2015). Mapping the diagnosis of autism spectrum disorders in children aged under 7 years in Australia, 2010-2012. Medical Journal of Australia, 202(6), 317-20. https://doi.org/10.5694/mja14.00328

Bialystok, E., Majumder, S., \& Martin, M. (2003). Developing phonological awareness: Is there a bilingual advantage? Applied Psycholinguistics, 24(1), 27-44. https://doi.org/10.1017/S014271640300002X

Brugha, T., Cooper, S., McManus, S., Purdon, S., Smith, J., Scott, F., Spiers, N., \& Tryer, F. (2012). Estimating the prevalence of Autism Spectrum Conditions in adults: extending the 2007 adult psychiatric morbidity survey. The NHS Information Centre for Health and Social Care, 1-31.

Buckley, S. (2005). Autism and Down Syndrome. Down Syndrome News and Update, 4(4), 114-120. https://doi.org/10.1542/gr.7-2-21

Canbay, O. (2011). Comparing the phonological awareness of bilingual and monolingual preschool children. Procedia - Social and Behavioral Sciences, 15, 976-80.

https://doi.org/10.1016/j.sbspro.2011.03.224

Carlson, S.M. \& Meltzoff, A. (2008). Bilingual experience and executive functioning in young children. Developmental Science, 11(2), 282-298.

Carnine, D. W., Silbert, J., Kame'enui, E. J., \& Tarver, S. G. (2004). Direct Instruction Reading. In Pearson Allyn Bacon Prentice Hal.

Caselli, M. C., Monaco, L., Trasciani, M., \& Vicari, S. (2008). Language in Italian Children With Down Syndrome and With Specific Language Impairment. Neuropsychology, 22(1), 2735. https://doi.org/10.1037/0894-4105.22.1.27

Castillo, H., Patterson, B., Hickey, F., Kinsman, A., Howard, J. M., Mitchell, T., \& Molloy, C. A. 
(2008). Difference in age at regression in children with autism with and without Down syndrome. Journal of Developmental and Behavioral Pediatrics, 29(2), 89-93. https://doi.org/10.1097/DBP.0b013e318165c78d

Chapman, R. S, \& Hesketh, L. J. (2000). Behavioral phenotype of individuals with Down syndrome. Mental Retardation and Developmental Disabilities Research Reviews, 6(2), 84-95. https://doi.org/AID-MRDD2>3.0.CO;2-P

Chapman, R. S., Seung, H. K., Schwartz, S. E., \& Kay-Raining Bird, E. (1998). Language skills of children and adolescents with Down syndrome: II. Production deficits. Journal of Speech, Language, and Hearing Research, 41(4), 861-73. https://doi.org/10.1044/jslhr.4104.861

Chen, X., Anderson, R. C., Li, W., Hao, M., Wu, X., \& Shu, H. (2004). Phonological Awareness of Bilingual and Monolingual Chinese Children. Journal of Educational Psychology, 96(1), 142-51. https://doi.org/10.1037/0022-0663.96.1.142

Christensen, D. L., Braun, K. V. N., Baio, J., Bilder, D., Charles, J., Constantino, J. N. ... YearginAllsopp, M. (2018). Prevalence and Characteristics of Autism Spectrum Disorder Among Children Aged 8 Years - Autism and Developmental Disabilities Monitoring Network, 11 Sites, United States, 2012. MMWR. Surveillance Summaries, 69(4), 1-12. https://doi.org/10.15585/mmwr.ss6513a1

Cleave, P. L., Kay-Raining Bird, E., Trudeau, N., \& Sutton, A. (2014). Syntactic bootstrapping in children with Down syndrome: The impact of bilingualism. Journal of Communication Disorders, 49, 42-54. https://doi.org/10.1016/j.jcomdis.2014.02.006

Crawford, J.R., \& Howell, D. C. (1998). Comparing an Individual's Test Score Against Norms Derived from Small Samples. The Clinical Neuropsychologist, 12(4), 482-86. https://doi.org/10.1076/clin.12.4.482.7241

Crawford, J. R., \& Garthwaite, P. H. (2005). Testing for suspected impairments and dissociations in single-case studies in neuropsychology: Evaluation of alternatives using Monte Carlo simulations and revised tests for dissociations. Neuropsychology, 19(3), 318-32. https://doi.org/10.1037/0894-4105.19.3.318

Crawford, J. R., \& Garthwaite, P. H. (2007). Comparison of a single case to a control or normative sample in neuropsychology: Development of a Bayesian approach. Cognitive Neuropsychology, 24(4), 343-372. https://doi.org/10.1080/02643290701290146

Crawford, J. R., Garthwaite, P. H., \& Gray, C. D. (2003). Wanted: Fully operational definitions of dissociations in single-case studies. In Cortex, 39(2), 357-70. https://doi.org/10.1016/S0010-9452(08)70117-5

Cupples, L., \& lacono, T. (2000). Phonological awareness and oral reading skill in children with Down syndrome. Journal of Speech, Language \& Hearing Research, 43(3), 595608.http://search.ebscohost.com/login. aspx?direct=true \&db=a3h\&AN=3226692\&site= ehost-live

de Bruin, A., Treccani, B., \& Della Sala, S. (2015). Cognitive Advantage in Bilingualism: An Example of Publication Bias? Psychological Science, 26(1), 99-107. https://doi.org/10.1177/0956797614557866

DiGuiseppi, C., Hepburn, S., Davis, J. M., Fidler, D. J., Hartway, S., Lee, N. R., Miller, L., 
Ruttenber, M., \& Robinson, C. (2010). Screening for Autism Spectrum Disorders in Children With Down Syndrome. Journal of Developmental \& Behavioral Pediatrics, 31(3), 181-191. https://doi.org/10.1097/DBP.0b013e3181d5aa6d

Drysdale, H., van der Meer, L., \& Kagohara, D. (2015). Children with Autism Spectrum Disorder from Bilingual Families: a Systematic Review. Review Journal of Autism and Developmental Disorders, 2(1), 26-38. https://doi.org/10.1007/s40489-014-0032-7

Feltmate, K., \& Kay-Raining Bird, E. (2008). Language learning in four bilingual children with Down syndrome: a detailed analysis of vocabulary and morphosyntax/L'apprentissage du langage chez quatre enfants bilingues atteints du syndrome de Down : une analyse detaillee du vocabulaire et de la morph. Canadian Journal of Speech-Language Pathology \& Audiology, 32(1), 6.

Fidler, D. J. (2005). The emerging down syndrome behavioral phenotype in early childhood: Implications for practice. In Infants and Young Children, 18(2), 86-103. https://doi.org/10.1097/00001163-200504000-00003

Fletcher, H., \& Buckley, S. (2002). Phonological awareness in children with Down syndrome. Down's Syndrome, Research and Practice: The Journal of the Sarah Duffen Centre / University of Portsmouth, 8(1), 11-18. https://doi.org/10.3104/reports.123

Gathercole, V. C., \& Thomas, E. M. (2007). Prawf Geirfa Cymraeg, Fersiwn 7-11. (Welsh Vocabulary Test, Version 7-11).

Godfrey, M., Hepburn, S., Fidler, D. J., Tapera, T., Zhang, F., Rosenberg, C. R., \& Raitano Lee, N. (2019). Autism spectrum disorder (ASD) symptom profiles of children with comorbid Down syndrome (DS) and ASD: A comparison with children with DS-only and ASD-only. Research in Developmental Disabilities, 89, 83-93. https://doi.org/10.1016/j.ridd.2019.03.003

Gonzalez-Barrero, A. M., \& Nadig, A. (2018). Bilingual children with autism spectrum disorders: The impact of amount of language exposure on vocabulary and morphological skills at school age. Autism Research, 11(12), 1667-1678. https://doi.org/10.1002/aur.2023

Hambly, C. \& Fombonne, E. (2012). The impact of bilingual environments on language development in children with autism spectrum disorders. Journal of Autism and Developmental Disorders, 42(7), 1342-1352.

Hampton, S., Rabagliati, H., Sorace, A., \& Fletcher-Watson, S. (2017). Autism and Bilingualism: A Qualitative Interview Study of Parents' Perspectives and Experiences. Journal of Speech, Language, and Hearing Research, 60(2), 435-446. https://doi.org/10.1044/2016_JSLHR-L-15-0348

Howard, K., Katsos, N., \& Gibson, J. L. (2019). The school experiences of bilingual children on the autism spectrum: An interpretative phenomenological analysis. Research in Developmental Disabilities, 87, 9-20. https://doi.org/10.1016/j.ridd.2019.01.008

Howard, K., Gibson, J., \& Katsos, N. (2020). Parental Perceptions and Decisions Regarding Maintaining Bilingualism in Autism. Journal of Autism and Developmental Disorders. https://doi.org/10.1007/s10803-020-04528-x

Jarrold, C., Baddeley, A. D., \& Hewes, A. K. (2000). Verbal Short-term Memory Deficits in 
Down Syndrome: A Consequence of Problems in Rehearsal? The Journal of Child Psychology and Psychiatry and Allied Disciplines, 41(2), 233-244.

https://doi.org/10.1017/S0021963099005120

Jensen, K. M., \& Bulova, P. D. (2014). Managing the care of adults with Down's syndrome. In BMJ (Online). https://doi.org/10.1136/bmj.g5596

Kaufman, A. S., \& Kaufman, N. L. (2004). Kaufman Brief Intelligence Test (2nd ed.). Bloomington, MN: Pearson, Inc.

Kay-Raining Bird, E., Cleave, P., Trudeau, N., Thordardottir, E., Sutton, A., \& Thorpe, A. (2005). The Language Abilities of Bilingual Children With Down Syndrome. American Journal of Speech-Language Pathology, 14(3), 187-199. https://doi.org/10.1044/10580360(2005/019)

Kay-Raining Bird, E., Lamond, E., \& Holden, J. (2012). Survey of bilingualism in autism spectrum disorders. International Journal of Language \& Communication Disorders / Royal College of Speech \& Language Therapists, 47(1), 52-64. https://doi.org/10.1111/j.1460-6984.2011.00071.x

Kennedy, E. J., \& Flynn, M. C. (2003). Early phonological awareness and reading skills in children with Down syndrome. Down's Syndrome, Research and Practice : The Journal of the Sarah Duffen Centre / University of Portsmouth, 8(3), 100-109. https://doi.org/10.3104/reports.136

Korbel, J. O., Tirosh-Wagner, T., Urban, A. E., Chen, X.-N., Kasowski, M., Dai, L., Grubert, F., Erdman, C. ... Korenberg, J. R. (2009). The genetic architecture of Down syndrome phenotypes revealed by high-resolution analysis of human segmental trisomies. Proceedings of the National Academy of Sciences, 106(29), 12031-6. https://doi.org/10.1073/pnas.0813248106

Kremer-Sadlik, T. (2005). To Be or Not to Be Bilingual: Autistic Children from Multilingual Families. ISB4: The 4th Annual Symposium on Bilingualism, 1225-1234.

Kroeger, K. A., \& Nelson, W. M. (2006). A language programme to increase the verbal production of a child dually diagnosed with Down syndrome and autism. Journal of Intellectual Disability Research, 50(2), 101-108. https://doi.org/10.1111/j.13652788.2005.00734.x

Kwok, E. Y. L., Brown, H. M., Smyth, R. E., \& Oram Cardy, J. (2015). Meta-analysis of receptive and expressive language skills in autism spectrum disorder. Research in Autism Spectrum Disorders, 9, 202-22. https://doi.org/10.1016/j.rasd.2014.10.008

Lanfranchi, S., Cornoldi, C., \& Vianello, R. (2004). Verbal and visuospatial working memory deficits in children with Down syndrome. American Journal on Mental Retardation, 109(6), 456-66. https://doi.org/10.1352/0895-8017(2004)109<456:VAVWMD>2.0.CO;2

Lehtonen, M., Soveri, A., Laine, A., Järvenpää, J., de Bruin, A., \& Antfolk, J. (2018). Is bilingualism associated with enhanced executive functioning in adults? A meta-analytic review. Psychological Bulletin, 144(4), 394-425. https://doi.org/10.1037/bul0000142

Loveland, K. A., Landry, S. H., Hughes, S. O., Hall, S. K., \& McEvoy, R. E. (1988). Speech acts and the pragmatic deficits of autism. Journal of Speech and Hearing Research, 31(4), 593-604. https://doi.org/10.1044/jshr.3104.593 
Marini, A., Sperindè, P., Ruta, I., Savegnago, C., \& Avanzini, F. (2019). Linguistic skills in bilingual children with developmental language disorders: A pilot study. Frontiers in Psychology. https://doi.org/10.3389/fpsyg.2019.00493

Marinova-Todd, S. H., Colozzo, P., Mirenda, P., Stahl, H., Kay-Raining Bird, E., Parkington, K., Cain, K., de Valenzuela, J. S., Segers, E., MacLeod, A. A. N., \& Genesee, F. (2016). Professional practices and opinions about services available to bilingual children with developmental disabilities: An international study. Journal of Communication Disorders, 63, 47-62. https://doi.org///dx.doi.org/10.1016/j.jcomdis.2016.05.004

Martin, G. E., Klusek, J., Estigarribia, B., \& Roberts, J. E. (2009). Language Characteristics of Individuals with Down Syndrome. Topics in Language Disorders, 29(2), 112-32.

Melby-Lervåg, M., Lyster, S. A. H., \& Hulme, C. (2012). Phonological skills and their role in learning to read: A meta-analytic review. Psychological Bulletin, 138(2), 322-52. https://doi.org/10.1037/a0026744

Molloy, C. A., Murray, D. S., Kinsman, A., Castillo, H., Mitchell, T., Hickey, F. J., \& Patterson, B. (2009). Differences in the clinical presentation of Trisomy 21 with and without autism. Journal of Intellectual Disability Research : JIDR, 53(2), 143-151. https://doi.org/10.1111/j.1365-2788.2008.01138.x

Morales, J., Calvo, A., \& Bialystok, E. (2013). Working memory development in monolingual and bilingual children. Journal of Experimental Child Psychology, 114(2), 187-202. https://doi.org/10.1016/j.jecp.2012.09.002

Morris, J.K., \& Alberman, E. (2009). Trends in Down's syndrome live births and antenatal diagnoses in England and Wales from 1989 to 2008: analysis of data from the National Down Syndrome Cytogenetic Register. The BMJ, 339. https://doi.org/doi.org/10.1136/bmj.b3794

Moss, J., Howlin, P., \& Oliver, C. (2012). The Assessment and Presentation of Autism Spectrum Disorder and Associated Characteristics in Individuals with Severe Intellectual Disability and Genetic Syndromes. In The Oxford Handbook of Intellectual Disability and Development. https://doi.org/10.1093/oxfordhb/9780195305012.013.0018

Neely, L., Gerow, S., Rispoli, M., Lang, R., \& Pullen, N. (2016). Treatment of Echolalia in Individuals with Autism Spectrum Disorder: a Systematic Review. Review Journal of Autism and Developmental Disorders, 3, 82-91. https://doi.org/10.1007/s40489-0150067-4

Papavassiliou, P., Charalsawadi, C., Rafferty, K., \& Jackson-Cook, C. (2015). Mosaicism for trisomy 21: A review. In American Journal of Medical Genetics, Part A, 167A(1), 26-39. https://doi.org/10.1002/ajmg.a.36861

Paradis, J., Crago, M., Genesee, F., \& Rice, M. (2003). French-English Bilingual Children With SLI: How Do They Compare With Their Monolingual Peers? Journal of Speech, Language, and Hearing Research, 46(1), 113-127. https://doi.org/10.1044/10924388(2003/009)

Parker, S.E., Mai, C.T., Canfield, M.A., Rickard, R., Wang, Y., Meyer, R.E., Anderson, P., Mason, C.A., ... Correa, A. (2010). Updated National Birth Prevalence estimates for selected birth defects in the United States, 2004-2006. Birth Defects Research, 88(12), 1008-1016. https://doi.org/doi: 10.1002/bdra.20735 
Parsons, L., Cordier, R., Munro, N., Joosten, A., \& Speyer, R. (2017). A systematic review of pragmatic language interventions for children with autism spectrum disorder. PLoS ONE, 12(4). https://doi.org/10.1371/journal.pone.0172242

Royal College of Speech and Language Therapists. (2019). Bilingualism - Key Guidance. 1-63.

Richards, C., Jones, C., Groves, L., Moss, J., \& Oliver, C. (2015). Prevalence of autism spectrum disorder phenomenology in genetic disorders: A systematic review and metaanalysis. The Lancet Psychiatry, 2(10), 909-16. https://doi.org/10.1016/S22150366(15)00376-4

Tager-Flusberg, H., \& Caronna, E. (2007). Language Disorders: Autism and Other Pervasive Developmental Disorders. In Pediatric Clinics of North America, 54(3), 469-481. https://doi.org/10.1016/j.pcl.2007.02.011

Tager-Flusberg, H., \& Kasari, C. (2013). Minimally verbal school-aged children with autism spectrum disorder: The neglected end of the spectrum. In Autism Research, 6(6), 46878. https://doi.org/10.1002/aur.1329

Uljarević, M., Katsos, N., Hudry, K., \& Gibson, J. L. (2016). Practitioner Review: Multilingualism and neurodevelopmental disorders - an overview of recent research and discussion of clinical implications. Journal of Child Psychology and Psychiatry, 57(11), 1205-1217. https://doi.org/10.1111/jcpp.12596

Valicenti-McDermott, M., Tarshis, N., Schouls, M., Galdston, M., Hottinger, K., Seijo, R., Shulman, L. \& Shinnar, S. (2013). Language differences between monolingual English and bilingual English-Spanish young children with autism spectrum disorders. Journal of Child Neurology, 28(7), 945-948.

van Bysterveldt, A. K., Gillon, G. T., \& Moran, C. (2006). Enhancing Phonological Awareness and Letter Knowledge in Preschool Children with Down Syndrome. International Journal of Disability, Development and Education, 53(3), 301-329. https://doi.org/10.1080/10349120600847706

Verhoeven, L. (2007). Early bilingualism, language transfer, and phonological awareness. Applied Psycholinguistics, 28, 425-439. https://doi.org/10.1017.S0142716407070233

von Bastian, C. C., Souza, A. S., \& Gade, M. (2016). No evidence for bilingual cognitive advantages: A test of four hypotheses. Journal of Experimental Psychology: General, 145(2), 246-258. https://doi.org/10.1037/xge0000120

Wang, M., Jegathesan, T., Young, E., Huber, J., \& Minhas, R. (2018). Raising children with autism spectrum disorders in monolingual vs bilingual homes: A scoping review. In Journal of Developmental and Behavioral Pediatrics, 39(5), 434-46. https://doi.org/10.1097/DBP.0000000000000574

Ware, J., Lye, C. B., \& Kyffin, F. (2015). Bilingualism and Students (Learners) With Intellectual Disability: A Review. Journal of Policy and Practice in Intellectual Disabilities, 12(3), 220-231. https://doi.org/10.1111/jppi.12124

Warner, G., Moss, J., Smith, P., \& Howlin, P. (2014). Autism characteristics and behavioural disturbances in 500 children with Down's syndrome in England and Wales. Autism Research, 7(4), 433-441. https://doi.org/10.1002/aur.1371

Welsh Government. (2020). No Schools by local authority, region and Welsh medium type. 
https://statswales.gov.wales/v/H-Ax

Wiig, E., Secord, W., \& Semel, E. (2006). The Clinical Evaluation of Language FundamentalsPreschool-2.

Ypsilanti, A., \& Grouios, G. (2008). Linguistic Profile of Individuals with Down Syndrome: Comparing the Linguistic Performance of Three Developmental Disorders. Child Neuropsychology, 14(2), 148-70. https://doi.org/10.1080/09297040701632209

$\mathrm{Yu}, \mathrm{B}$. (2013). Issues in bilingualism and heritage language maintenance: perspectives of minority-language mothers of children with autism spectrum disorders. American Journal of Speech-Language Pathology / American Speech-Language-Hearing Association, 22, 10-24. https://doi.org/10.1044/1058-0360(2012/10-0078)a

Zablotsky, B., Black, L.I., Maenner, M.J., Schieve, L.A., \& Blumberg, S. (2015). Estimated Prevalence of Autism and Other Developmental Disabilities Following Questionnaire Changes in the 2014 National Health Interview Survey. National Health Statistics Reports, 87(1), 1-20.

Zhao, W., Chen, F., Wu, M., Jiang, S., Wu, B., Luo, H., ... Yu, S. (2015). Postnatal identification of trisomy 21: An overview of 7,133 postnatal trisomy 21 cases identified in a diagnostic reference laboratory in China. PLOS ONE, 10(7). https://doi.org/10.1371/journal.pone.0133151 
Tables

Table 1.

Demographic characteristics for each case study participant.

Note: SES = Parental socioeconomic status. *Indicates missing information.

Table 2.

Demographic characteristics for the control group participants.

Note: Mean scores are reported with SD in parenthesis. Data is missing for 5 children from the BTD group for SES, parent-reported current exposure to Welsh and parent-reported lifetime exposure to Welsh due to non-return of the parental questionnaire. Age of first word missing data for 15 participants due to non-return of questionnaire or parents not being able to recall this information.

${ }^{a}$ Indicates a between-group effect of diagnosis with $p<.05$.

${ }^{b}$ Indicates a between-group effect of language status with $p<.05$.

Table 3.

Order of test administration for all participants. 


\section{Table 4.}

Dylan's performance on all measures in comparison to the control groups which shows any significant effects with point estimates and 95\% confidence intervals. Raw scores are reported with SD in parenthesis for between group comparisons.

Note: Point estimates indicates the percentage of the population in the control group estimated to perform below case participant. Bold indicates significant effects with $p<.05$.

\section{Table 5.}

Catrin's performance on all measures in comparison to the control groups which shows any significant effects with point estimates and $95 \%$ confidence intervals. Raw scores are reported with SD in parenthesis for between group comparisons.

Note: Point estimates indicates the percentage of the population in the control group estimated to perform below case participant. Bold indicates significant effects with $p<.05$.

\section{Table 6.}

Owain's performance on all measures in comparison to the control groups which shows any significant effects with point estimates and 95\% confidence intervals. Raw scores are reported with SD in parenthesis for between group comparisons.

Note: Point estimates indicates the percentage of the population in the control group estimated to perform below case participant. Bold indicates significant effects with $p<.05$. 
Table 7.

Rhiannon's performance on all measures in comparison to the control groups which shows any significant effects with point estimates and 95\% confidence intervals. Raw scores are reported with SD in parenthesis for between group comparisons.

Note: Point estimates indicates the percentage of the population in the control group estimated to perform below case participant. Bold indicates significant effects with $p<.05$. 